\title{
Study on Vibration and Noise For the Hydraulic System of Hydraulic hoist
}

\author{
Ning Chenxiao \\ College of Mechanical and Engineering Hebei University of \\ Science and Technology, \\ Shijiazhuang Hebei China \\ ningchenxiao@163.com
}

\begin{abstract}
By analysis on all kinds of the vibration source and noise mechanism, this paper points out the vibration and noise harm, cause and source of the hydraulic hoist hydraulic system. Further more it puts forward to effective and specific measures to reduce the vibration and noise of the hydraulic hoist hydraulic system which can also be widely applied to vibration and noise control of other hydraulic system.
\end{abstract}

Keywords-Hydraulic system; Vibration; Noise; Control measures

\section{PREFACE}

Hydraulic hoist used for hydraulic gate hoist is the important equipment in Large scale water conservancy. The vibration and noise of hydraulic hoist hydraulic system can effect the working performance of the hydraulic system and components, reduce the service life of the equipment and components, which affects the scure and reliable operation of the gate opening and closing system. At the same time, the noise cause irritability, fatigue, impact on the health of the people $^{[1]}$ 。 Therefore, it is very necessary to research on vibration and noise causes of the hydraulic system and puts forward the effective measures of reducing noise and vibration.

\section{VIBRATION AND NOISE SOURCE ANALYSIS OF HYDRAULIC SYSTEM OF HYDRAULIC HOIST}

Vibration is the inherent attribute of elastic body, which causes the hydraulic system noise. Vibration will generate the corresponding noise. Hydraulic impact, cavitations, the unbalanced force of mechanism rotates, moving components of the friction and inertia force changes are vibration and noise source ${ }^{[2]}$. The hydraulic system of hydraulic headstock gear vibration and noise comes mainly from two aspects: mechanical vibration and noise and fluid vibration and noise.

\section{A. Mechanical Vibration and Noise}

\section{1) Structural impact and Friction}

Bad processing and assembling precision of the components, which resulting in a mechanical stop stiffening or uneven friction resistance, cause vibration and noise, such as concentricity between the piston or piston rod and the cylinder concentricity, surface galling of the cylinder or piston rod in the inner wall.

\author{
Zhang Xushe \\ College of Mechanical and Engineering Hebei University of \\ Science and Technology, \\ Shijiazhuang Hebei China
}

\section{2) Rotary balance}

When motor, hydraulic pump, hydraulic motors in hydraulic system are rotating at a high speed, the rotating part imbalance can result in periodic unbalanced force, incentive mechanical vibration and then cause noise.

\section{3) Coupling}

Coupling the processing precision and the connection assembly precision, determines the pump shaft and a prime mover driven shaft coaxial degree.If this value out-of-tolerance, then vibration and noise caused.

\section{4) Hydraulic valve}

The valve core of overflow valve, electromagnetic valve, one-way valve generally supported in the spring, which is very sensitive to the vibration. Improper assembly will cause noise and vibration. Pressure regulating hand wheel loosening, deformation of the spring, damping hole plug valve hole, cylindrical spool and coaxial error of the relief valve can also cause noise and vibration.

5) Hydraulic cylinder

Hydraulic cylinder in fast reversing and large variation of load condition will cause hydraulic impact, thereby produce vibration and noise.

\section{6) Pipeline and Tank}

Pipeline and tank is not the source of vibration. The vibration is influenced by other components caused by vibration, such as pressure and flow pulsation, mechanical vibration and so on. When natural frequencies and vibration frequency of the pipeline and tank is same, then resonance occurs, resulting in strong noise. Especially when pipeline is too slender or meticulous and direction changes too much, more easily to cause vibration and noise.

\section{B. Fluid Vibration and Noise}

1) Hydraulic fluid pump vibration and noise

The main noise source in hydraulic equipment is the hydraulic pump. Flow pulsation of hydraulic pumps is its inherent characteristics, which is bound to cause the pump outlet pressure pulsation and piping, and to spread to the whole system, at the same time to generate vibration and noise. In addition, the gear pump packeted oil phenomena, the backward 
flow of plunger pump, the hydraulic pump leakage and pump hysteresis are also important factors of noise and vibration.

\section{2) Hydraulic valve vibration and noise}

When passes through the orifice or other valve port the oil fluid becomes high-speed jet,then partial pressure decreased sharply which leads to vibration and noise. Most of those from the overflow valve and a throttle valve are high pressure fluid which causes shear flow, turbulence or vortex in the jet with the surrounding fluid, resulting in high frequency vibration and noise.

\section{3) Hydraulic impact}

When the speed is high and the load inertia is large, sudden opening and closing valve or reversing can make the liquid inside the system produce hydraulic impact. When the pressure peak is too large it causes not only the great vibration and noise but so the hydraulic system damage.

\section{4) Cavitation induced vibration and noise}

There is about $2 \% \sim 5 \%$ air mixed with the hydraulic oil, so during the hydraulic system work it is easy to produce cavitation phenomenon in hydraulic pump suction port and orifice or narrow gap. Cavitation occurs, bubbles with the oil flowed into a high-pressure zone will produce bigger local instantaneous burst, pressure fluctuation, making the system produce vibration and noise. Cavitation also caused normal oil volume drop and resulted in slow motion components poor response.

Blocking of the suck oil filter and oil inlet and too high hydraulic oil viscosity can caused high degree of vacuum in the pump inlet place and make the air infiltration easily. When pump suction pipe is inserted into the fuel tank oil too short and oil suction pipe layup and pump shaft seal is lax, air is easy to be sucked into the pump. Air into the hydraulic system, will certainly cause noise and vibration.

\section{5) Turbulent flow and vortex}

Due to high flow velocity, flow cross section mutation, the liquid flow will be in the turbulent state and generates a local eddy. When there is turbulent flow and vortex, fluid particle motion, mutual impact, and interaction with the oil pump, cylinder, particle, pipeline, valve body wall or other interactions will produce vibration and noise.

\section{CONTROL MEASURES OF HOIST HydraUlic SySTEM VIBRATION AND NOISE}

In principle, there are two ways to control of hoist hydraulic system vibration and noise: one is to prevent and reduce vibration and noise generation; two is to prevent and reduce vibration and noise transmission. Based on the analysis of noise sources, to meet the needs of other functions in the system and to satisfy the overall economic feasible basis, available measures can be taken as following.

\section{A. System Component Selection}

\section{1) Motor and coupling.}

Selection of stable operation, low noise motor, and the choice of elastic coupling.

\section{2) Hydraulic pump}

Select small pulsating flow pump. Flow pulsation of the pump from is screw pump, vane pump, gear pump and plunger pump.

\section{3) Directional valve}

O type, $\mathrm{M}$ type a function valve with high precision, but the $\mathrm{O}$ type, $\mathrm{M}$ type and $\mathrm{Y}$ type reversing valve reversing bad stationarity, are easy to generate impact vibration. In order to reduce the impact of change, when the flow rate is less than the $60 \mathrm{~L} / \mathrm{min}$ commutation circuit, should preferre the DC electromagnet driving reversing valve. Direct acting relief valve has the advantages of simple structure, but easy to produce noise and vibration, so it is suitable for low pressure and small flow occasions; a pilot type overflow valve commutation impact of small, stable pressure, suitable for high pressure, large flow.

\section{4) Connection}

Hydraulic valve should better use plate connection or plugin type connection and use hydraulic integrated block instead of pipeline, in order to reduce the vibration.

\section{5) Parameter selection}

Experiments show that the noise of hydraulic pump with hydraulic power increases. Hydraulic power and pump working pressure $\mathrm{P}, \mathrm{V}$ and displacement speed is proportional to $\mathrm{N}$, but $\mathrm{p}, \mathrm{V}, \mathrm{N}$ on the vibration of the pump noise affected to different degrees, in which the speed of the pump $n$ has the most significant influence. Visible, low speed and large displacement is good for vibration reduction.

\section{B. To prevent cavitation induced vibration and noise}

- The system shall be provided in the air exhaust device, exhaust system in the air.

- To reduce the pump suction height; to select suitable oil filter; to make the pump suction pipe to rough enough, to make oil suction pipe and the pressure pipe diameter generally from 1.7 to 2.4; low temperature, oil viscosity increase, pumps prone to oil shortage phenomenon, should be based on seasonal temperature choose different viscosity of hydraulic oil, or by preheating measures of. All those are measures to improve the pump suction condition as far as possible.

- $\quad$ The overhead type fuel tank, reasonable set of baffles in the oil tank and a sufficient depth of the oil suction pipe inserted into the fluid, reliable joint of the oil suction pipe and sealing of shaft seal should be ensure to prevente air infiltration.

- When began to use the station stored for a long time must use the exhaust device fully exhaust or use with load after period of non-load running again.

\section{To prevent the hydraulic shock caused by vibration and noise}

- Extension of the valve opening and closing and moving parts brake reversing time, limiting pipeline fluid velocity and the velocity of moving parts. 
- Settings in the system of accumulator, the damping hose, to increase the system flexibility ${ }^{[3]}$.

- The buffer device is arranged in the hydraulic cylinder.

- $\quad$ Set back pressure valve, which can prevent overshoot phenomenon and impact load.

\section{Prevent system flow pressure pulsation induced vibration and noise}

- When design the hydraulic pump, pump module of gear try to take small, number of teeth try to take more; plunger of plunger pump a number should be an odd number, usually $7 \sim 9$, and in the swash plate provided on symmetric triangular grooves, to prevent the plunger pump oil.

- Add energy accumulator in the system to absorb pressure pulsation and flow, because the accumulator can absorb the noise below $10 \mathrm{~Hz}$.

- We can connect rubber hose near the hydraulic pump oil outlet because rubber hose to absorb high frequency noise is very effective.

- Ripple reduction device tandem connected in the pipeline ( pulsation damper ), can eliminate the pipeline flow pulsation.

\section{E. To prevent the pipeline and tank vibration and noise}

- The length of the pipe should be as short as possible, swerved should be less curved, a circular should be transited with arc. The metal hard tube, pipe layout should be parallel to each other and to leave a gap. Select support frame interval according to the diameter size and add rubber pads or wood pad between the oil pipe and support frame to damping.

- Reinforcing tank rigid, set isolation board to control the vibration and noise of the oil tank.

- When the pump's motor, hydraulic pump and the fuel tank share the same base, should installate shock absorber under the motor base, to avoid the mechanical vibration to a tank.

F. Take the isolation and sound insulation method to control the vibration and noise of the outside path.

- Install antivibration rubber pad on the motor, hydraulic pump and the hydraulic valve installation surface.
- Cover hydraulic pump with a sound absorbing material sound insulation cover, which can effectively reduce the noise.

\section{THE END}

Vibration and noise of Hydraulic hoist hydraulic system is more complex, not only the unbalanced force, inertia force, friction resistance caused by a variety of mechanical vibration and noise, but also and the pressure and flow pulsation, cavitation, hydraulic shock caused by different types of fluid vibration and noise.According to different sources, measures should be taken from the hydraulic system design, manufacturing, installation, using and to adopt a reasonable reduction and other aspects to control the vibration and noise generate and transmit to a minimum range.Vibration and noise of hydraulic system generating mechanism is similar, these measures can also be widely used in various types of hydraulic system of vibration and noise reduction.

\section{REFERENCES}

[1] Zhang Zhong-shi, "Study on vibration and noise of hydraulic system," Joumal of Inner Mongolia Agricultural University, vol.29, No.1, pp.130-133, Mar 2008.

[2] Gao Jiu-hao, Gong Lie-hang, Li Qi, "Reasons and resolutions for vibration and noise of the metal fluid vessels," Machine Tool \& Hydraulics, 2005.No.4, pp.189-196.

[3] Wang Xing, Huang Zhi-jian, He Xiang-hua, "Diagnosis of vibration in the hydraulic system for rolling mill,” Southern Metals, June 2010, pp.8-10.

[4] Li Jian-yu, "Analysis for the Reason of Producing Vibration Noise of Hydraulic System,” Chinese Hydraulic \& Pneumatics, 2006. No.5, pp.76-78.

[5] Wu Li-qun, Yang Yi-mei, "Analysis the reason of vibration and yawp of hydraulic system,” Mechanical Research \& Application, vol.19, No.5, pp.15-16, October 2006.

[6] Song Da-qing, "Absorbing Vibration and Noise in Hydraulic System,” Fluid Power Transmission and Control, Mar 2007. No.3, Serial No.22, pp.49-50.

[7] Han Quanli, Wang Hongying, "Prevention and Improvement Measures for the Vibration and Noise of the Hydraulic System of a Numerical Control Machine,” Mechanical Science and Technology for Aerospace Engineering, vol. 29, No.8, pp.1110-1111, August 2010.

[8] Zhang lin, "The analysis of the quiver and the noises of the hydraulic pressure system,” Mechanical Research \& Application, vol.18, No.6, pp.43, December 2005. 